\title{
EXPERIENCES WITH CANADIAN ALTASWEDE AND SWEDISH COMMERCIAL RED CLOVER IN FINLAND
}

\author{
OtTo Valle \\ Agricultural Research Centre, Department of Plant Husbandry, \\ Tikkurila, Finland
}

Received October 31, 1958.

For several years the cultivation of red clover in Finland has been dependent to a great extent on imported seed. Prior to 1950 Sweden was the primary source of this seed. More recently there have been several years when seed of the Swedish single-cut, later flowering type was not available in sufficient quantities to supply the need for it in Finland. When this situation occurred Canadian Altaswede was used, a single-cut variety earlier in flowering habit than Finnish red clover.

The first Altaswede seed - a shipment of 200 tons - was imported by Finland in 1948. During the six-year period 1953-1958 an average of 226 tons of Altaswede seed was brought into the country annually. This represented 80 percent of the total red clover seed imported by Finland during the period. Of the balance, 13 percent was Swedish commercial and 7 percent was from the U.S.S.R. During the years 1953, 1957 and 1958 Sweden was not able to export any single-cut red clover seed to Finland (1).

The first trials comparing Altaswede and Finnish Tammisto red clover, the first Finnish bred variety, were arranged in Finland in 1948. The trials were at seven different experimental stations from Southern Finland to the Artic Circle. The results of the trials planted in 1948-1950 were published in 1953 (2) and are summarized in Table 1. In first year leys the Altaswede was 8 percent lower in yield than the Finnish Tammisto red clover; in second year ley it was 21 percent lower. These results are collected in Table 1. Significance is estimated by the difference method using Fisher's $t$-table: * signif. $95 \%$; ** signif. $99 \%$; *** signif. $99.9 \%$ 
Table 1. Results from red clover variety trials (Tammisto, Altaswede) in Finland. Planting years 1948 - 50.

\begin{tabular}{|c|c|c|c|c|c|c|}
\hline \multirow{2}{*}{$\begin{array}{c}\text { Year } \\
\text { of } \\
\text { planting }\end{array}$} & \multirow{2}{*}{ Variety } & \multicolumn{2}{|c|}{$\begin{array}{l}\text { Winter survival } \\
10-0\end{array}$} & \multicolumn{3}{|c|}{$\begin{array}{c}\text { Dry matter yield } \\
\text { Tammisto } \mathrm{kg} / \text { hectare }=100\end{array}$} \\
\hline & & $\begin{array}{c}\text { lst } \\
\text { winter }\end{array}$ & $\begin{array}{c}\text { 2nd } \\
\text { winter }\end{array}$ & $\begin{array}{c}\text { lst } \\
\text { year }\end{array}$ & $\begin{array}{l}2 \mathrm{nd} \\
\text { year }\end{array}$ & Average \\
\hline
\end{tabular}

Agricultural Research Centre, Department of Plant Husbandry, Tikkurila, latit. $60^{\circ} 18^{\prime}$

\begin{tabular}{|c|c|c|c|c|c|c|}
\hline \multirow[t]{3}{*}{1948} & Tammisto & 9.4 & 6.5 & 6950 & 8020 & 7490 \\
\hline & & & & 100 & 100 & 100 \\
\hline & Altaswede & 7.9 & 2.5 & 90 & 67 & 78 \\
\hline \multirow[t]{3}{*}{$-n-$} & Tammisto & 9.2 & 7.0 & 6780 & 8700 & 7740 \\
\hline & & & & 100 & 100 & 100 \\
\hline & Altaswede & 8.4 & 3.8 & 100 & 76 & 86 \\
\hline \multirow[t]{3}{*}{1949} & Tammisto & 9.5 & 9.8 & 5810 & 7040 & 6430 \\
\hline & & & & 100 & 100 & 100 \\
\hline & Altaswede & 9.2 & 9.4 & 102 & 79 & 89 \\
\hline \multirow[t]{3}{*}{1950} & Tammisto & 6.4 & 7.9 & 3940 & 3160 & 3550 \\
\hline & & & & 100 & 100 & 100 \\
\hline & Altaswede & 6.8 & 7.0 & 109 & 88 & 9 \\
\hline
\end{tabular}

Tammisto Plant Breeding Station, Helsingin pit., latit. $60^{\circ} 17^{\prime}$

$\begin{array}{rrrrrrr}1948 & \text { Tammisto } & 9.5 & 9.9 & 7060 & 6870 & 6970 \\ & & & & 100 & 100 & 100 \\ & \text { Altaswede } & 9.0 & 9.7 & 93 & 91 & 92 \\ 1950 & & & & 2940 & 7910 & 5430 \\ & \text { Tammisto } & 9.9 & 9.8 & 100 & 100 & 100 \\ & & & & 99 & 96 & 98\end{array}$

Agricultural Research Centre, Department of Plant Breeding, Jokioinen, latit. $60^{\circ} 49^{\prime}$

\begin{tabular}{|c|c|c|c|c|c|c|}
\hline \multirow[t]{2}{*}{1948} & Tammisto & 7.6 & 9.7 & 7900 & 5100 & 6500 \\
\hline & & & & 100 & 100 & 100 \\
\hline \multirow{4}{*}{1949} & Altaswede & 7.1 & 9.3 & 91 & 70 & 83 \\
\hline & Tammisto & 9.8 & 9.8 & 5560 & 3610 & 4590 \\
\hline & & & & 100 & 100 & 100 \\
\hline & Altaswede & 10.0 & 9.8 & 97 & 70 & \\
\hline
\end{tabular}

South Ostrobothnia Agricultural Experiment Station, Ylistaro, latit. $62^{\circ} 57^{\prime}$

\begin{tabular}{|c|c|c|c|c|c|c|}
\hline \multirow[t]{3}{*}{1948} & Tammisto & 6.0 & 5.5 & 8550 & 5250 & 6900 \\
\hline & & & & 100 & 100 & 100 \\
\hline & Altaswede & 3.0 & 2.2 & 62 & 61 & 62 \\
\hline
\end{tabular}

Pasture Experiment Station, Mouhijärvi, latit. $61^{\circ} 31^{\prime}$

$\begin{array}{rrrrrrr}1948 & \text { Tammisto } & 8.4 & 8.8 & 6720 & 5610 & 6170 \\ & & & 100 & 100 & 100 \\ & \text { Altaswede } & 7.0 & 6.6 & 83 & 59 & 72\end{array}$

North Ostrobothnia Agricultural Experiment Station, Ruukki, latit. $64^{\circ} \mathbf{4 1}$

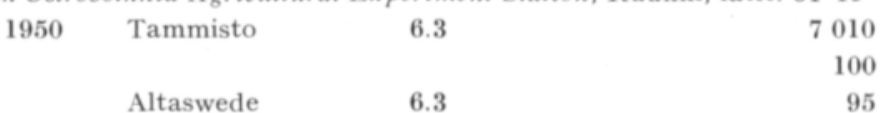




\begin{tabular}{|c|c|c|c|c|c|}
\hline \multirow{2}{*}{$\begin{array}{c}\text { Year } \\
\text { of } \\
\text { planting }\end{array}$} & \multirow{2}{*}{ Variety } & $\begin{array}{l}\text { Winter survival } \\
10-0\end{array}$ & \multicolumn{3}{|c|}{$\begin{array}{c}\text { Dry matter yield } \\
\text { Tammisto } \mathrm{kg} / \text { hectare }=100\end{array}$} \\
\hline & & $\begin{array}{c}\text { 2nd } \\
\text { winter }\end{array}$ & $\begin{array}{c}\text { lst } \\
\text { year }\end{array}$ & $\begin{array}{l}\text { 2nd } \\
\text { year }\end{array}$ & Average \\
\hline
\end{tabular}

Polar Circle Agricultural Experiment Station, Apukka, latit. $66^{\circ} 35^{\prime}$

\begin{tabular}{|c|c|c|c|c|c|c|}
\hline \multirow[t]{3}{*}{1948} & Tammisto & 9.0 & 8.0 & 3440 & 4000 & 3720 \\
\hline & & & & 100 & 100 & 100 \\
\hline & Altaswede & 8.0 & 6.5 & 93 & 76 & 84 \\
\hline \multirow[t]{3}{*}{1949} & Tammisto & 9.3 & 9.3 & 7290 & 1990 & 4640 \\
\hline & & & & 100 & 100 & 100 \\
\hline & Altaswede & 7.3 & 6. 7 & 88 & 118 & 94 \\
\hline \multirow{4}{*}{$\begin{array}{l}\text { rage } \\
948-50\end{array}$} & & & & & & \\
\hline & Tammisto & 8.5 & 8.5 & 6150 & 5610 & 5840 \\
\hline & & & & 100 & 100 & 100 \\
\hline & Altaswede & 7.7 & 6.9 & 92 & 79 & 85 \\
\hline
\end{tabular}

$$
\begin{gathered}
\text { Significance in dry matter yield } \\
\text { Ist year } \\
\text { Tammisto-Altaswede } 580 \pm 252^{*} \mathrm{~kg} / \mathrm{ha} \\
2 n d \text { year } \\
\text { Tammisto-Altaswede } 1120 \pm 333^{* *} \mathrm{~kg} / \mathrm{ha}
\end{gathered}
$$

\begin{tabular}{|c|c|c|c|c|c|c|c|}
\hline \multirow{2}{*}{$\begin{array}{c}\text { Year } \\
\text { of } \\
\text { planting }\end{array}$} & \multirow{2}{*}{$\begin{array}{l}\text { Variety or } \\
\text { origin }\end{array}$} & $\begin{array}{l}\text { Winter survival } \\
10-0\end{array}$ & \multicolumn{2}{|c|}{ Cutting dates } & \multicolumn{3}{|c|}{$\begin{array}{c}\text { Dry matter yield } \\
\text { Tammisto } \mathrm{kg} / \text { hectare }=100\end{array}$} \\
\hline & & $\begin{array}{c}\text { lst } \quad \text { nd } \\
\text { winter winter }\end{array}$ & $\begin{array}{c}\text { lst } \\
\text { year }\end{array}$ & $\begin{array}{l}\text { 2nd } \\
\text { year }\end{array}$ & $\begin{array}{c}\text { lst } \\
\text { year }\end{array}$ & $\begin{array}{l}\text { 2nd } \\
\text { year }\end{array}$ & Average \\
\hline
\end{tabular}

\begin{tabular}{|c|c|c|c|c|c|c|c|c|}
\hline \multirow[t]{3}{*}{1952} & Tammisto & 2.8 & 7.4 & $1 / 7,7 / 8$ & $10 / 7, \quad 7 / 9$ & 4010 & 4510 & 4260 \\
\hline & Altaswede & 1.8 & 5.0 & & & 74 & 56 & 65 \\
\hline & Swedish commercial & 1.8 & 6.0 & & & 83 & 78 & 81 \\
\hline \multirow[t]{3}{*}{1953} & Tammisto & & 8.2 & $7 / 7,26 / 8$ & $20 / 7, \quad 14 / 9$ & & 6030 & 6030 \\
\hline & Altaswede & & 3.6 & & & & 63 & 63 \\
\hline & Swedish commercial & & 6.3 & & & & 82 & 82 \\
\hline \multirow[t]{3}{*}{1954} & Tammisto & 9.7 & 8.9 & $18 / 7, \quad 9 / 9$ & $4 / 7,28 / 8$ & 7680 & 7170 & 7430 \\
\hline & Altaswede & 9.7 & 7.8 & & & 96 & 82 & 89 \\
\hline & Swedish commercial & 9.7 & 8.3 & & & 83 & 88 & 86 \\
\hline \multirow[t]{3}{*}{1955} & Tammisto & 4.3 & & $9 / 7,24 / 8$ & & 4460 & & 4460 \\
\hline & Altaswede & 4.6 & & & & 91 & & 91 \\
\hline & Swedish commercial & 1.6 & & & & 87 & & 87 \\
\hline \multirow[t]{3}{*}{1956} & Tammisto & 9.5 & & $2 / 8,17 / 9$ & & 9920 & & 9920 \\
\hline & Altaswede & 7.5 & & & & 77 & & 77 \\
\hline & Swedish commercial & 9.0 & & & & 77 & & 77 \\
\hline \multicolumn{9}{|c|}{ Tammisto Plant Breeding Station, Helsingin pit., latit. $60^{\circ} 17^{\prime}$} \\
\hline \multirow[t]{3}{*}{1953} & Tammisto & 7.8 & 8.7 & $7 / 7,19 / 8$ & $19 / 7$ & 5800 & 4480 & 5140 \\
\hline & Altaswede & 5.9 & 4.3 & & & 85 & 46 & 66 \\
\hline & Swedish commercial & 5.5 & 6.0 & & & 83 & 54 & 69 \\
\hline
\end{tabular}

Table 2. Results from red clover variety trials (Tammisto, Altaswede, Swedish commercial) in Finland. Planting years $1952-56$. 


\begin{tabular}{|c|c|c|c|c|c|c|c|c|}
\hline \multirow{2}{*}{$\begin{array}{c}\text { Year } \\
\text { of } \\
\text { planting }\end{array}$} & \multirow{2}{*}{$\begin{array}{l}\text { Variety or } \\
\text { origin }\end{array}$} & \multicolumn{2}{|c|}{$\begin{array}{c}\text { Winter survival } \\
10-0\end{array}$} & \multicolumn{2}{|c|}{ Cutting dates } & \multicolumn{3}{|c|}{$\begin{array}{c}\text { Dry matter yield } \\
\text { Tammisto } \mathrm{kg} / \text { hectare }=100\end{array}$} \\
\hline & & $\begin{array}{c}\text { lst } \\
\text { winter }\end{array}$ & $\begin{array}{c}\text { 2nd } \\
\text { winter }\end{array}$ & $\begin{array}{c}\text { lst } \\
\text { year }\end{array}$ & $\begin{array}{l}\text { 2nd } \\
\text { year }\end{array}$ & $\begin{array}{c}\text { lst } \\
\text { year }\end{array}$ & $\begin{array}{l}\text { 2nd } \\
\text { year }\end{array}$ & Average \\
\hline \multirow[t]{3}{*}{1955} & Tammisto & 6.0 & & $16 / 7,30 / 8$ & & 2520 & & 2520 \\
\hline & Altaswede & 3.0 & & & & 57 & & 57 \\
\hline & Swedish commercial & 5.9 & & & & 115 & & 115 \\
\hline \multicolumn{9}{|c|}{ Agricultural Research Centre, Department of Plant Breeding, Jokioinen, latit. $60^{\circ} 49^{\prime}$} \\
\hline \multirow[t]{3}{*}{1953} & Tammiste & 4.3 & 6.2 & $16 / 7,21 / 9$ & $14 / 7$ & 6830 & 1980 & 4410 \\
\hline & Altaswede & 2.3 & 1.6 & & & 77 & 32 & 55 \\
\hline & Swedish commercial & 3.3 & 2.4 & & & 78 & 45 & 62 \\
\hline \multirow[t]{3}{*}{1955} & Tammisto & 9.0 & 7.2 & $6 / 7,24 / 8$ & $8 / 7,10 / 9$ & 4720 & 9410 & 7060 \\
\hline & Altaswede & 8.5 & 5.8 & & & 90 & 86 & 88 \\
\hline & Swedish commercial & 8.4 & 5.4 & & & 110 & 73 & 92 \\
\hline \multirow[t]{3}{*}{1956} & Tammisto & 7.3 & & $9 / 7, \quad 9 / 9$ & & 11400 & & 11400 \\
\hline & Altaswede & 7.0 & & & & 85 & & 85 \\
\hline & Swedish commercial & 7.4 & & & & 98 & & 98 \\
\hline \multicolumn{9}{|c|}{ Fiskars Farm, Pohja, latit. $60^{\circ} 06^{\prime}$} \\
\hline \multirow[t]{3}{*}{1954} & Tammisto & 8.5 & 9.1 & $13 / 7$ & $10 / 7,28 / 8$ & 5620 & 6320 & 5970 \\
\hline & Altaswede & 7.5 & 8.1 & & & 90 & 94 & 92 \\
\hline & Swedish commercial & 8.0 & 8.8 & & & 88 & 93 & 91 \\
\hline \multirow[t]{3}{*}{1955} & Tammisto & 5.5 & 4.4 & $12 / 7,29 / 8$ & $8 / 7, \quad 4 / 9$ & 3630 & 3960 & 3800 \\
\hline & Altaswede & 5.5 & 1.8 & & & 105 & 88 & 97 \\
\hline & Swedish commercial & 6.2 & 3.7 & & & 111 & 92 & 102 \\
\hline \multirow[t]{3}{*}{$-\cdots$} & Tammisto & 6.0 & & $10 / 7,28 / 8$ & & 6230 & & 6230 \\
\hline & Altaswede & 4.8 & & & & 84 & & 84 \\
\hline & Swedish commercial & 6.0 & & & & 103 & & 103 \\
\hline \multicolumn{9}{|c|}{ Carelia Agricultural Experiment Station, Anjala, latit. $60^{\circ} 42^{\prime}$} \\
\hline \multirow[t]{3}{*}{1955} & Tammisto & 4.6 & & $13 / 7,20 / 9$ & & 6460 & & 6460 \\
\hline & Altaswede & 1.7 & & & & 69 & & 69 \\
\hline & Swedish commercial & 2.5 & & & & 93 & & 93 \\
\hline \multicolumn{9}{|c|}{ Häme Agricultural Experiment Station, Pälkäne, latit. $61^{\circ} 20^{\prime}$} \\
\hline \multirow[t]{3}{*}{1956} & Tammisto & 8.8 & & $4 / 7, \quad 4 / 9$ & & 9820 & & 9820 \\
\hline & Altaswede & 9.4 & & & & 94 & & 94 \\
\hline & Swedish commercial & 7.9 & & & & 67 & & 67 \\
\hline \multicolumn{9}{|c|}{ South Ostrobothnia Agricultural Experiment Station, Ylistaro, latit. $62^{\circ} 57^{\prime}$} \\
\hline \multirow[t]{3}{*}{1953} & Tammisto & 7.1 & 4.6 & $5 / 8$ & $9 / 8$ & 8000 & 6560 & 7280 \\
\hline & Altaswede & 3.6 & 2.3 & & & 78 & 82 & 80 \\
\hline & Swedish commercial & 6.2 & 2.7 & & & 104 & 94 & 99 \\
\hline 1956 & Tammisto & 9.3 & & $6 / 8$ & & 11250 & & 11250 \\
\hline & Altaswede & 8.0 & & & & 86 & & 86 \\
\hline & Swedish commercial & 8.6 & & & & 97 & & 97 \\
\hline North Sa: & wo Agricultural Experis & nent Stat. & ion, Maz & aninka, latit. & $33^{\circ} 09^{\prime}$ & & & 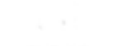 \\
\hline 1954 & Tammisto & 4.4 & 2.8 & $29 / 7$ & $16 / 7$ & 5820 & 6860 & 6340 \\
\hline & Altaswede & 1.4 & 0.6 & & & 86 & 70 & 78 \\
\hline & Swedish commercial & 3.9 & 1.5 & & & 101 & 85 & 93 \\
\hline 1955 & Tammisto & 4.6 & 8.8 & $12 / 7$ & $29 / 7$ & 5280 & 8010 & 6640 \\
\hline & Altaswede & 2.4 & 6.4 & & & 87 & 81 & 84 \\
\hline & Swedish commercial & 4.3 & 8.1 & & & 87 & 88 & 88 \\
\hline 1956 & Tammisto & 7.8 & & $30 / 7$ & & 7470 & & 7470 \\
\hline & Altaswede & 5.5 & & & & 81 & & 81 \\
\hline & Swedish commercial & 4.5 & & & & 62 & & 62 \\
\hline
\end{tabular}




\begin{tabular}{|c|c|c|c|c|c|c|}
\hline \multirow{2}{*}{$\begin{array}{c}\text { Year } \\
\text { of } \\
\text { planting }\end{array}$} & \multirow{2}{*}{$\begin{array}{l}\text { Variety or } \\
\text { origin }\end{array}$} & $\begin{array}{c}\text { Winter survival } \\
10-0\end{array}$ & \multicolumn{2}{|c|}{ Cutting dates } & \multicolumn{2}{|c|}{$\begin{array}{c}\text { Dry matter yield } \\
\text { Tammisto } \mathrm{kg} / \text { hectare }=100\end{array}$} \\
\hline & & $\begin{array}{c}\text { lst } \quad \text { 2nd } \\
\text { winter winter }\end{array}$ & $\begin{array}{l}\text { lst } \\
\text { year }\end{array}$ & $\begin{array}{l}\text { 2nd } \\
\text { year }\end{array}$ & $\begin{array}{c}\text { lst } \\
\text { year }\end{array}$ & $\begin{array}{l}\text { 2nd } \\
\text { year }\end{array}$ \\
\hline
\end{tabular}

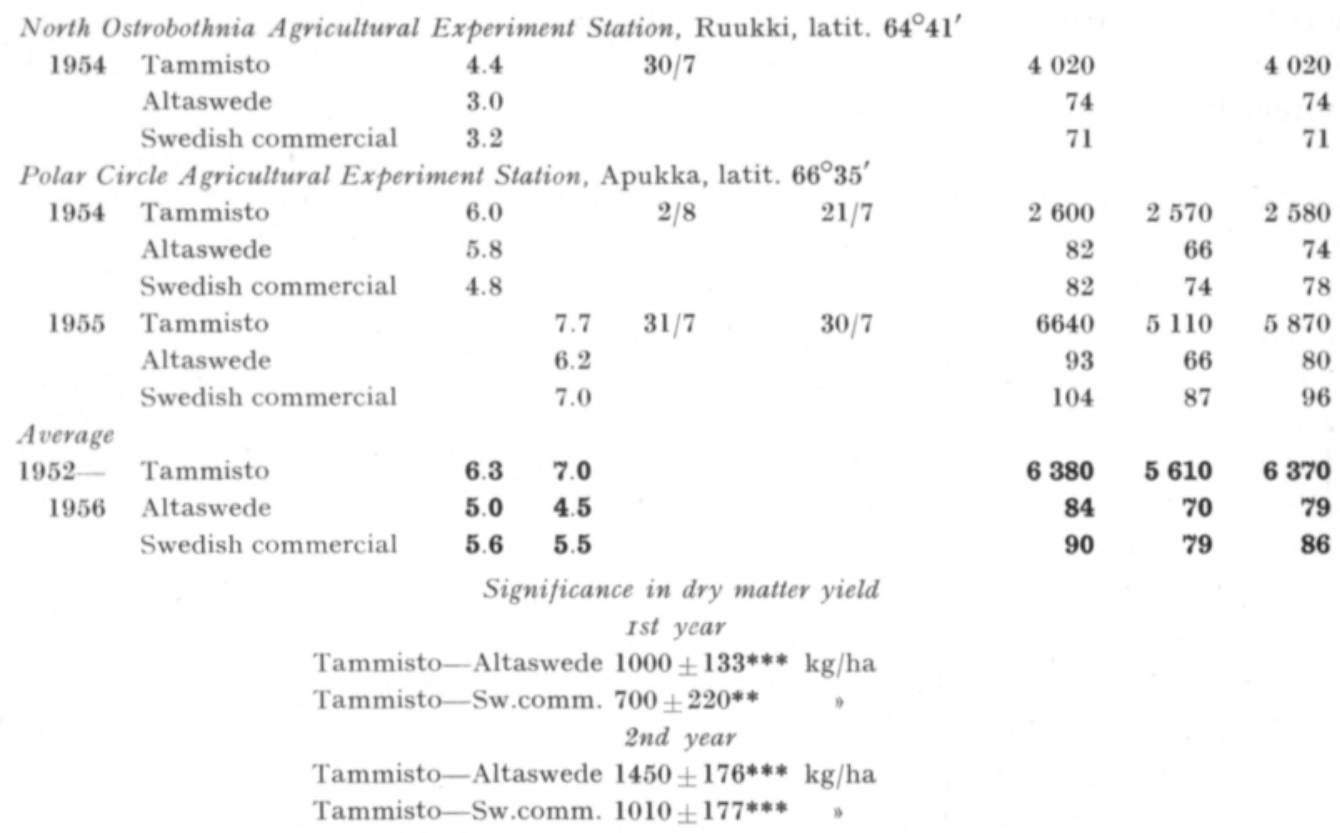

In 1953, after a lapse of five years, Altaswede seed was imported again, at which time new trials were started in Finland. Since then trials have been planted each year. These have been at 10 experimental sites, the northernmost at the Polar Circle Agricultural Experiment Station (latitude $66^{\circ} 35^{\prime}$ ). The most extensive tests have been located at the Agricultural Research Centre, Department of Plant Husbandry, Tikkurila in Southern Finland. Tests established at the different experimental sites during each of the past five years included Finnish Tammisto red clover as the standard, Altaswede and imported Swedish commercial red clover, representing the single-cut late flowering type.

The plot size has been 10 square meters and usually included 5 replications. A greater part of the trials were planted with a companion crop.

The tests in Southern Finland were cut twice during the growing season; the main crop during the early flowering stage, and the regrowth at the end of August or in September. The tests in Middle and Northern Finland were harvested once each season usually during the last part of July or early August. At most locations the clover was harvested for two seasons.

Winter survival for the different clovers was estimated at the beginning of each growing season. A scale of $10-0$ was used with 10 representing no injury and 0 complete killing. In addition to the dry matter yields the amount of crude protein in the forage was determined for some of the trials. The results from 22 trials conducted during 1952-1956 are given in Table 2. 
In the trials during the $1952-1956$ period winter survival in the first and second year leys averaged 6.3 and 7.0 for Tammisto, 5.0 and 4.5 for Altaswede, and 5.6 and 5.5 for Swedish commercial, respectively. The difference in winter survival in these trials between Altaswede and Tammisto was much greater than in earlier trials because of the several severe winters for red clover in the 1950's. This can be noted by comparing the results given in Tables 1 and 2 . Thus the results on winter survival from all trials show conclusively that the injury to Altaswede has been extremely heavy during the second winter.

Winter survival of the Swedish commercial red clover has been surprisingly poor. Data show it to be more comparable to Altaswede than to Tammisto in winter survival although the extent of the differences varied between trials.

Differences in forage yields have been significant among the three sources of red clover seed tested. The dry matter yield of Tammisto red clover averaged, in first year leys, 6.380 kilos per hectare (5620 lbs/acre) and in second year leys 5.610 kilos per hectare (4940 lbs/acre). In comparing the dry matter yield of first year leys, Altaswede and Swedish commercial were $16 \%$ and $10 \%$ lower, respectively than Tammisto. In second year leys the difference in yields was even more pronounced for Altaswede yielded 30 percent less and Swedish commercial 21 percent less. When the total yields for the first and second year leys are taken into account the yield of Altaswede was 21 percent lower and Swedish commercial 16 percent lower than the Finnish bred Tammisto variety.

Red clover is the most important legume plant in Finland, and it cannot be replaced satisfactorily by other species such as alfalfa. Thus in years following a short red clover seed crop in Finland it has been necessary to import red clover seed for forage production.

The results from the Finnish red clover trials indicate that the Canadian Altaswede and Swedish commercial red clover are not equal in winter survival and in forage yield to the Finnish Tammisto red clover.

For this reason seed production of the single-cut Finnish Tammisto red clover has been started abroad. Tammisto was ready for the market in 1937, but red clover seed production has been erratic in Finland due to the humid and rainy weather especially during harvest.

The experiences in producing seed of Tammisto red clover have been most satisfactory on the North American Continent - both in Canada and the United States. It is the plan to replace in the very near future the Canadian Altaswede and the Swedish commercial with North American-grown red clover seed of the Finnish bred Tammisto variety, to insure protein production in Finland (3).

\section{R E F E R E N C E S}

(1) VAlle, O. 1957. The problem of red clover seed production in Finland. Maatal. tiet. aikak. 29: 177-184. Helsinki.

(2) $\longrightarrow$ 1953. Kokemuksia kanadalaisesta Altaswede-puna-apilasta. (Finnish). Koetoim. ja käyt. 4: 1. Helsinki.

(3) - 1958. North America produces red clover seed for Finland. Foreign Agriculture (U.S.) Vol. XXI, 12: 13--14. 
S E L O T U S :

SUOMESSA SAATUJA KOKEMUKSIA KANADAN ALTASWEDESTA JA RUOTSALAISESTA PUNA-APILASTA

Otto Valle

Maatalouden tutkimuskeskuksen Kasvinviljelylaitos, Tikkurila

Tähän kirjoitukseen on koottu tuloksia Suomessa suoritetuista puna-apilan kantakokeista, joissa Tammiston puna-apilaa on verrattu kanadalaiseen Altaswedeen ja ruotsalaiseen kauppasiemeneen.

Vuosina 1948 - 50 perustetuissa kokeissa antoi Altaswede 1. vuoden nurmessa $8 \%$ ja 2. vuoden nurmessa $21 \%$ heikomman kuiva-ainesadon kuin Tammisto (taul. 1). Vuosina 1952-56 kylvetyissä kokeissa oli taas 1. vuoden nurmessa Altaswede $16 \%$ ja ruotsalainen puna-apila $10 \%$ Tammistoa heikkosatoisempi vastaavien satoerojen ollessa 2. vuoden nurmessa Tammiston hyväksi $30 \%$ ja $21 \%$ (taul. 2).

Tähänastinen kanadalainen tai ruotsalainen tuontisiemen olisi mahdollisimman pian korvattava Tammiston puna-apilalla, jonka siementä ei Suomessa ole saatu lisätyksi, mutta jonka siemenviljelykokeilut ovat ulkomailla käynnissä.

\title{
MAATALOUSTIETEELLINEN AIKAKAUSKIRJA TOIMITUSKUNTA
}

Erkki Kivinen, Antti Mäki, Vilho A. Pesola, Ilmari Poijärvi, Onni Pohjakallio

\author{
E. A. Jamalainen \\ Toimitussihteeri
}

Tikkurila. Puh. 831419 ja 831318

MAATALOUSTIETEELLISTA AIKAKAUSKIRJAA

ilmestyy 4 vihkoa vuodessa

Käsikirjoitukset lähetetään toimitussihteerille (os. Tikkurila) tai jollekin toimitusvaliokunnan jäsenelle

\section{SUOMEN MAATALOUSTIETEELLINEN SEURA}

Puheenjohtaja:

Professori Juhani Paatela

Kulosaarent. 21, Helsinki

Varapuheenjohtaja:

Maat. ja metsät. toht. Paavo Kajanoja

Ratak. 1 a B. Helsinki
Sihteeri:

Professori V. Vainikainen

Kasarmik. 38 A 8, Helsinki

Rahastonhoitaja:

Tohtori Orvo Ring

Tikkurila

Kirjastonhoitaja:

Maisteri Majlis Tulander, Hallituskatu 3, Helsinki 